\title{
Estudo comparativo da mortalidade infantil no estado do Rio Grande do Sul e suas mesorregiões
}

\author{
Comparative study of infant mortality in the state of Rio Grande do Sul \\ and its mesoregions
}

\author{
Jéssica Rodrigues da Silva', Ana Lúcia Souza Silva Mateus", \\ Augusto Maciel da Silva "I
}

\begin{abstract}
RESUMO
O número de óbitos infantis vem diminuindo ao longo dos anos no mundo. O intuito deste artigo é identificar o cenário da mortalidade infantil, no Rio Grande do Sul, verificando se o mesmo se reflete em suas mesorregiões. Para isso, foram realizadas técnicas de estatística descritiva, de correlação com o teste de Spearman e de componentes principais. Os resultados obtidos na análise descritiva revelaram que em geral o Rio Grande do Sul e suas mesorregiões se comportam da mesma forma, as correlações se mostraram altas entre as variáveis, exceto com a variável, proporção de mulheres que realizaram menos de 7 consultas pré-natais. A análise de componentes principais também mostrou que as variáveis das mesorregiões e do estado são explicadas por duas componentes. Com isso, conclui-se que o cenário da mortalidade infantil se reflete em suas mesorregiões.
\end{abstract}

Palavras-chave: Óbitos Infantis; Sistema de Informação; Análise de Componentes Principais.

\begin{abstract}
The number of child deaths has been decreasing over the years, in the world, in Brazil and in Rio Grande do Sul. The intuition of this article is to identify the scenario of infant mortality in Rio Grande do Sul, verifying if it is reflected in its mesoregions. For this, descriptive statistics, correlation with Spearmam test and principal components techniques were performed. The results obtained in the descriptive analysis revealed that in general Rio Grande do Sul and its mesoregions behave the same way, the correlations were high among the variables, except with the variable, proportion of women who attended less than 7 prenatal consultations. Principal component analysis also showed that the mesoregion and state variables are explained by two components. Thus, it is concluded that in fact the scenario of infant mortality is reflected in its mesoregions.
\end{abstract}

Keywords: Infant Deaths; Information Systems; Analysis of Principal Components. 


\section{INTRODUÇÃO}

A taxa de mortalidade infantil (TMI) mede o risco de morte de crianças menores de um ano de idade sendo considerada como um dos mais importantes indicadores de qualidade de vida de uma população (VERMELHO et al., 2006), expressando, assim, os níveis de saúde e grau de desenvolvimento de uma sociedade (ANDRADE et al., 2006).

A mortalidade infantil vem diminuindo nos países desenvolvidos desde o século XX, em virtude das melhores condições de vida e saneamento das populações. A partir da década de 60, avanços tecnológicos na área da saúde e programas recomendados pela Organização Mundial da Saúde (OMS), contribuíram para uma forte redução na mortalidade de crianças no primeiro ano de vida (OLIVEIRA e MENDES, 1990).

A Cúpula do Milênio das Nações Unidas em 2000 estabeleceram metas para melhorar a qualidade das pessoas, os chamados Objetivos de Desenvolvimento do Milênio (ODMs) que se resumem em oito objetivos internacionais de desenvolvimento para o ano de 2015. De acordo com o relatório divulgado pela Organização das Nações Unidas o Brasil conseguiu reduzir significativamente a mortalidade infantil, atingindo o Objetivo do Milênio 4 (ODM 4), reduzindo de 61\% em 1990 para 16\% em 2015, uma redução de $73 \%$ (ONU, 2105).

Conforme dados do Instituto Brasileiro de Geografia e Estatística (IBGE, 2013), da mesma forma que no Brasil, no Rio grande do Sul também se observou uma redução na mortalidade infantil. Em 1990 a TMI foi de 26,2 óbitos por 1000 nascidos vivos, enquanto em 2011 foi de 11,1 óbitos por 1000 nascidos vivos. Reduzindo em 58\% a TMI em 21 anos (1990 - 2011). Porém, as TMl's no Rio Grande do Sul sempre se apresentaram menores que as brasileiras, se tornando mais próximas nos últimos anos.

Mesmo com essa tendência decrescente, a mortalidade infantil é uma das principais preocupações dos governos em programas de saúde pública, pois constitui um dos indicadores de avaliação de qualidade de vida. De modo consequente, alguns autores questionam o real valor da taxa de mortalidade como indicador de qualidade de vida (AHMAD, et al. 2000; CLAESON, et al. 2000). 
Estudos sobre a mortalidade infantil têm sido descritos por diversas técnicas: regressão sequencial de Poisson (HERNANDEZ et al., 2011), teste qui-quadrado de associação (BRUM et al.,2014), curva ROC (Receiver Operator Characteristic) (LOOSE et al., 2014), estatística espacial (SHIMAKURA et al., 2001; SCALON et al., 2012) e regressão logística multivariada (GEIB et al., 2010; NASCIMENTO et al., 2012; SANTOS et al., 2012).

Segundo Escofier e Pages (1992), os métodos de análise de dados multivariados são muito eficazes em estudos com grande volume de informações e complexidade. As finalidades de aplicação da técnica são as mais diversas, como de reduzir a dimensionalidade dos dados ou de simplificação estrutural, de classificar e agrupar, de investigar a dependência entre variáveis, de predição e de elaborar hipóteses e testálas (JOHNSON; WICHERN, 1992). Desta forma, este artigo tem por objetivo, analisar o comportamento da mortalidade infantil no estado do Rio Grande do Sul utilizando técnicas de estatística descritiva, de correlação com o teste de Spearman e de componentes principais. O intuito é de identificar quais as variáveis são determinantes no número de óbitos infantis dentro do estado e verificar se este comportamento reflete nas suas mesorregiões.

\section{MATERIAL E MÉTODOS}

O local de estudo é o estado do Rio Grande do Sul e suas mesorregiões. Localizado ao sul do Brasil, o estado, apresenta uma extensão de $281.731,445$ km², 497 municípios e 11.286 .500 habitantes. Ele é divido geograficamente em sete mesorregiões, sendo: Centro Ocidental, Centro Oriental, Metropolitana, Nordeste, Noroeste, Sudeste e Sudoeste, cada uma com 31, 54, 98, 54, 217, 25 e 18 municípios, respectivamente.

Avaliou-se um total de 10 variáveis: o número de óbitos em crianças menores de um ano de idade (OI); baixo peso ao nascer $(<2500 \mathrm{~g})$ - (BP); duração gestacional $(<37$ semanas) - (DG); mulheres em idade reprodutiva (15 a 49 anos) - (MIR); parto cesariano - (PC); parto vaginal - (PV); variáveis relacionadas ao sexo do bebê: feminino - (F) e masculino - (M); óbitos ocorridos na primeira semana de vida (0 a 7 dias) - (S1) e 
proporção de mulheres que realizaram menos de 7consultas pré-natais (C6), por setor censitário (município).

Para as análises foi considerado o somatório das ocorrências de cada variável nos anos de 2009 a 2013 por município, para o estado e suas mesorregiões. Sendo a variável C6 proveniente do Sistema de Informação de Nascidos Vivos (SINASC) e as remanescentes, do Sistema de Informações sobre Mortalidade (SIM). Ambos os sistemas oriundos do Departamento de Informática do Sistema Único de Saúde do Brasil (DATASUS). Foram excluídos os registros cadastrados no sistema como ignorados. Foi realizada inicialmente uma análise descritiva dos dados, visando ter maior conhecimento de suas variações. Em seguida, análise de correlação, com o intuito de identificar o grau de relação entre as variáveis e por fim a análise de componentes principais com intuito de identificar as inter-relações entre as variáveis de estudo.

$\mathrm{Na}$ análise de correlação utilizou-se abordagem não paramétrica de Spearman. Segundo FERREIRA (2005), o Coeficiente de Correlação de Spearman $\left(r_{s}\right)$ exige que as duas variáveis se apresentem em escala de mensuração pelo menos ordinal, de forma que os elementos (indivíduos ou objetos) em estudo formem duas séries ordenadas. Para verificar se há correlação entre as variáveis, é realizado o teste de correlação, em que, a hipótese nula é que não existe correlação significativa entre as variáveis contra a hipótese alternativa de que existe correlação significativa. A classificação do coeficiente de correlação foi realizada conforme Dancey e Reidy (2005) sendo $r_{s}=0,0$ até 0,30 (fraco); $r_{s}=0,40$ até 0,6 (moderado); $r_{s}=0,70$ até 1 (forte).

A análise de componentes principais consiste essencialmente em reescrever as coordenadas das amostras em outro sistema de eixo mais conveniente para a análise dos dados. Em outras palavras, as n-variáveis originais geram, através de suas combinações lineares, n-componentes principais, cuja principal característica, além da ortogonalidade, é que são obtidos em ordem decrescente de máxima variância, ou seja, a componente principal 1 detém mais informação estatística que a componente principal 2, que por sua vez tem mais informação estatística que a componente principal 3 e assim por diante. Está análise também pode ser usada para julgar a 
importância das próprias variáveis originais escolhidas, ou seja, as variáveis originais com maior peso (loadings) na combinação linear dos primeiros componentes principais são as mais importantes do ponto de vista estatístico (MOITA NETO; MOITA, 1998)

Neste artigo a análise de componentes principais foi feita via matriz de correlação. Maiores detalhes sobre o procedimento matemático para obtenção de componentes principais pode ser encontrado em Ferreira (2011). As análises foram realizadas no software R (R Development Core Team, 2015). Para as análises de correlação, foram fixados dois valores críticos para o nível de significância ( $\alpha$ ), de 1\% e $5 \%$.

\section{RESULTADOS}

\subsection{Análise descritiva}

Com os resultados obtidos na análise descritiva, verificou-se que há uma grande discrepância dentro das variáveis analisadas, conforme pode ser observado pelos valores dos coeficientes de variação. Isso acontece tanto no estado como nas mesorregiões, estes resultados estão apresentados no Quadro 1.

Quadro 1 - Análises descritivas das variáveis em estudo no estado do Rio Grande do Sul e suas mesorregiões

\begin{tabular}{|c|c|c|c|c|c|c|c|c|c|c|c|}
\hline & Medidas & $\mathrm{OI}$ & $\mathrm{BP}$ & DG & MIR & PV & PC & C6 & $\mathrm{M}$ & $\mathrm{F}$ & S1 \\
\hline \multirow{6}{*}{$\begin{array}{l}\text { Rio } \\
\text { Grande } \\
\text { do Sul }\end{array}$} & Média & 15,3099 & 9,7586 & 9,5453 & 14,4648 & 6,497 & 8,4145 & 0,2155 & 8,3662 & 6,8873 & 7,6499 \\
\hline & Mediana & 3 & 2 & 2 & 3 & 1 & 2 & 0,2 & 2 & 1 & 2 \\
\hline & $\begin{array}{l}\text { Desvio } \\
\text { Padrão }\end{array}$ & 53,2771 & 35,9496 & 34,8081 & 51,7589 & 24,4461 & 28,5944 & 0,1065 & 29,5468 & 23,7718 & 25,0701 \\
\hline & Mínimo & 0 & 0 & 0 & 0 & 0 & 0 & 0 & 0 & 0 & 0 \\
\hline & Máximo & 913 & 621 & 595 & 897 & 433 & 474 & 0,566 & 509 & 402 & 413 \\
\hline & $\begin{array}{c}\text { Coeficiente } \\
\text { de } \\
\text { Variação }\end{array}$ & 347,9923 & 368,3912 & 364,6632 & 357,8266 & 376,2678 & 339,8232 & 49,4496 & 353,1683 & 345,1534 & 327,7181 \\
\hline \multirow{6}{*}{$\begin{array}{l}\text { Centro } \\
\text { Ocidental }\end{array}$} & Média & 11,6129 & 7,2581 & 6,9355 & 11,0323 & 4,7742 & 6,6129 & 0,2486 & 6,3226 & 5,1935 & 4,9032 \\
\hline & Mediana & 3 & 2 & 2 & 2 & 1 & 2 & 0,2299 & 1 & 2 & 1 \\
\hline & $\begin{array}{l}\text { Desvio } \\
\text { Padrão }\end{array}$ & 32,9329 & 23,245 & 22,1735 & 31,8282 & 14,4861 & 17,9864 & 0,0958 & 19,083 & 13,6977 & 13,1107 \\
\hline & Mínimo & 0 & 0 & 0 & 0 & 0 & 0 & 0,0876 & 0 & 0 & 0 \\
\hline & Máximo & 184 & 130 & 124 & 178 & 81 & 100 & 0,5602 & 106 & 76 & 71 \\
\hline & $\begin{array}{c}\text { Coeficiente } \\
\text { de } \\
\text { Variação }\end{array}$ & 283,5892 & 320,2648 & 319,7104 & 288,5009 & 303,4252 & 271,99 & 38,5283 & 301,8222 & 263,7452 & 267,3892 \\
\hline \multirow{3}{*}{$\begin{array}{l}\text { Centro } \\
\text { Oriental }\end{array}$} & Média & 8,8148 & 5,7222 & 5,6667 & 8,2778 & 3,4444 & 4,963 & 0,1936 & 4,8148 & 3,9815 & 3,8704 \\
\hline & Mediana & 3 & 1,5 & 2 & 2,5 & 1 & 2 & 0,201 & 2 & 1 & 1 \\
\hline & $\begin{array}{l}\text { Desvio } \\
\text { Padrão }\end{array}$ & 14,455 & 9,9724 & 9,6837 & 13,8406 & 6,2031 & 7,8907 & 0,0814 & 7,9148 & 6,8308 & 6,1584 \\
\hline
\end{tabular}




\begin{tabular}{|c|c|c|c|c|c|c|c|c|c|c|c|}
\hline & Mínimo & 0 & 0 & 0 & 0 & 0 & 0 & 0,0222 & 0 & 0 & 0 \\
\hline & Máximo & 64 & 44 & 44 & 63 & 26 & 37 & 0,4557 & 30 & 35 & 24 \\
\hline & $\begin{array}{c}\text { Coeficiente } \\
\text { de } \\
\text { Variação }\end{array}$ & 163,985 & 174,2758 & 170,8884 & 167,2022 & 180,0889 & 158,9912 & 42,0522 & 164,3846 & 171,5646 & 159,1174 \\
\hline \multirow{6}{*}{$\begin{array}{l}\text { Metro- } \\
\text { politana }\end{array}$} & Média & 33,6327 & 21,3367 & 20,8367 & 31,8878 & 15,2755 & 17,6531 & 0,2365 & 18,2959 & 15,1735 & 16,1122 \\
\hline & Mediana & 8 & 4 & 4 & 7,5 & 4 & 3,5 & 0,243 & 4 & 3 & 3 \\
\hline & $\begin{array}{l}\text { Desvio } \\
\text { Padrão }\end{array}$ & 100,8648 & 67,8055 & 65,2264 & 98,6065 & 47,9026 & 52,3663 & 0,0971 & 55,9943 & 44,8339 & 46,1477 \\
\hline & Mínimo & 0 & 0 & 0 & 0 & 0 & 0 & 0 & 0 & 0 & 0 \\
\hline & Máximo & 913 & 621 & 595 & 897 & 433 & 474 & 0,5342 & 509 & 402 & 413 \\
\hline & $\begin{array}{c}\text { Coeficiente } \\
\text { de } \\
\text { Variação } \\
\end{array}$ & 299,9013 & 317,7876 & 313,0358 & 309,2299 & 313,5906 & 296,6414 & 41,0515 & 306,0482 & 295,4759 & 286,4135 \\
\hline \multirow{6}{*}{ Nordeste } & Média & 14,5185 & 9,6852 & 9,5185 & 13,6667 & 5,2222 & 8,8148 & 0,1767 & 7,8704 & 6,6296 & 7,3889 \\
\hline & Mediana & 2 & 1 & 1 & 2 & 1 & 1 & 0,1564 & 1 & 1 & 1 \\
\hline & $\begin{array}{l}\text { Desvio } \\
\text { Padrão }\end{array}$ & 50,4357 & 36,8714 & 35,6808 & 48,6877 & 19,116 & 30,7737 & 0,1059 & 26,8174 & 23,6765 & 24,9395 \\
\hline & Mínimo & 0 & 0 & 0 & 0 & 0 & 0 & 0,0405 & 0 & 0 & 0 \\
\hline & Máximo & 364 & 267 & 258 & 352 & 138 & 221 & 0,5102 & 194 & 170 & 179 \\
\hline & $\begin{array}{c}\text { Coeficiente } \\
\text { de } \\
\text { Variação }\end{array}$ & 347,389 & 380,6993 & 374,8571 & 356,2513 & 366,051 & 349,1136 & 59,9082 & 340,7384 & 357,1317 & 337,5269 \\
\hline \multirow{6}{*}{ Noroeste } & Média & 5,8571 & 3,7189 & 3,5806 & 5,553 & 2,2535 & 3,4931 & 0,1906 & 3,1889 & 2,6544 & 3,2857 \\
\hline & Mediana & 2 & 1 & 1 & 2 & 1 & 1 & 0,1713 & 1 & 1 & 1 \\
\hline & $\begin{array}{l}\text { Desvio } \\
\text { Padrão }\end{array}$ & 14,3638 & 9,3768 & 9,0786 & 13,914 & 5,9532 & 8,4525 & 0,1006 & 7,6149 & 6,9339 & 8,0813 \\
\hline & Mínimo & 0 & 0 & 0 & 0 & 0 & 0 & 0 & 0 & 0 & 0 \\
\hline & Máximo & 152 & 98 & 99 & 149 & 64 & 86 & 0,566 & 79 & 73 & 83 \\
\hline & $\begin{array}{c}\text { Coeficiente } \\
\text { de } \\
\text { Variação }\end{array}$ & 245,2348 & 252,1407 & 253,5458 & 250,568 & 264,179 & 241,9793 & 52,7745 & 238,7921 & 261,2253 & 245,9516 \\
\hline \multirow{6}{*}{ Sudoeste } & Média & 37,9444 & 25,1111 & 24,7778 & 36 & 18,9444 & 18,2222 & 0,359 & 21,5556 & 16,2778 & 21,2778 \\
\hline & Mediana & 20,5 & 10 & 11 & 20 & 9,5 & 9,5 & 0,3636 & 11,5 & 8,5 & 13,5 \\
\hline & $\begin{array}{l}\text { Desvio } \\
\text { Padrão }\end{array}$ & 46,7377 & 31,6002 & 31,4447 & 44,5131 & 23,7176 & 22,2143 & 0,1253 & 27,184 & 20,1197 & 24,5424 \\
\hline & Mínimo & 0 & 0 & 0 & 0 & 0 & 0 & 0,1592 & 0 & 0 & 0 \\
\hline & Máximo & 183 & 123 & 120 & 174 & 92 & 87 & 0,5555 & 105 & 78 & 95 \\
\hline & $\begin{array}{c}\text { Coeficiente } \\
\text { de } \\
\text { Variação }\end{array}$ & 81,186 & 79,4649 & 78,7979 & 80,8752 & 79,8749 & 82,0291 & 286,4329 & 79,2951 & 80,9048 & 86,698 \\
\hline \multirow{6}{*}{ Sudeste } & Média & 30,24 & 18,24 & 18,2 & 28,48 & 11,96 & 17,36 & 0,3045 & 16,48 & 13,68 & 14,92 \\
\hline & Mediana & 11 & 6 & 5 & 10 & 4 & 8 & 0,304 & 5 & 7 & 5 \\
\hline & $\begin{array}{l}\text { Desvio } \\
\text { Padrão }\end{array}$ & 61,7976 & 39,4771 & 40,4125 & 58,2681 & 23,5062 & 37,9516 & 0,068 & 35,7189 & 26,1131 & 31,6556 \\
\hline & Mínimo & 0 & 0 & 0 & 0 & 0 & 0 & 0,1636 & 0 & 0 & 0 \\
\hline & Máximo & 276 & 181 & 186 & 257 & 88 & 180 & 0,4724 & 161 & 115 & 146 \\
\hline & $\begin{array}{c}\text { Coeficiente } \\
\text { de } \\
\text { Variação }\end{array}$ & 204,3571 & 216,4314 & 222,0465 & 204,5932 & 196,5399 & 218,6152 & 22,3397 & 216,7412 & 190,8851 & 212,1689 \\
\hline
\end{tabular}

OI: óbitos infantis; BP: baixo peso ao nascer (<2500 g); DG: duração gestacional (<37 semanas); MIR: mulheres em idade reprodutiva (15 a 49 anos); PV: parto vaginal; PC: parto cesariano; C6: proporção de mulheres que realizaram menos de 7 consultas pré-natais; M: variável relacionada ao sexo masculino; F: variável relacionada ao sexo feminino e S1: óbitos na primeira semana de vida. Observações válidas nas demais tabelas. 
Pelo Quadro 1, observa-se que em média, todas as variáveis estão com altos índices de ocorrência na mesorregião Sudoeste, assim como, os menores índices de ocorrência de todas as variáveis estão na mesorregião Noroeste. Com exceção da variável C6, onde a menor média encontra-se na mesorregião Nordeste.

Todas as variáveis em todas as mesorregiões possuem seus valores mínimos de ocorrência iguais a zero, exceto a variável C6, que apresenta na mesorregião Sudeste (Encruzilhada do Sul) seu valor mínimo de 0,1636. Em média, os valores máximos de ocorrência encontram-se em todas as variáveis. A se destacar na mesorregião Metropolitana no município de Porto Alegre e na mesorregião Noroeste no município de Santo Antônio das Missões, onde a variável C6 foi de 0,566.

O Ministério da Saúde preconiza ao menos seis consultas de pré-natal (BRASIL, 2000). Em média, a proporção de mulheres que realizaram menos de 7 consultas prénatais é baixa para o estado e suas mesorregiões. Nunes et al. (2017) realizaram um estudo transversal descritivo sobre a Pesquisa Nacional de Saúde em 2013 no Brasil, concluíram que a proporção de mulheres que afirmaram haver realizado pré-natal no Brasil foi de $97,4 \%$. E que, por mais que a região Sul tenha apresentado uma ampla cobertura do acompanhamento do pré-natal, a mesma ainda é a segunda menor região com cobertura de $96,3 \%$ e o estado do Rio Grande do Sul com 88,20\%.

Avaliando o coeficiente de variação (CV), temos que todas as variáveis têm menor dispersão na mesorregião Sudoeste, ao mesmo tempo em que, todas as variáveis têm maior dispersão na mesorregião Nordeste, ainda com exceção da variável C6, que apresenta maior dispersão na mesorregião Sudoeste.

Observou-se também que, tanto no estado do Rio Grande do Sul quanto em suas mesorregiões, o número de óbitos de bebês do sexo masculino é superior ao número de óbitos de bebês do sexo feminino. Devido ao fato dos bebês do sexo feminino apresentar um amadurecimento pulmonar mais precoce, estudos descrevem que a variável sexo masculino é fortemente determinante nas mortes infantis no primeiro ano de vida (MENEZES et al., 2005; ARAÚjO et al., 2000). Quanto aos partos existem uma superioridade dos partos cesarianos sobre os vaginais, no estado e em todas as mesorregiões, exceto na mesorregião Sudoeste, onde o número de cesáreas é inferior. 


\subsection{Análise de Correlação}

Na Tabela 1, observa-se que no estado do Rio Grande do Sul e na mesorregião Noroeste, encontraram-se correlações entre as variáveis estudadas e o número de óbitos em sua grande maioria forte, sendo todas significativas pelo Teste de Correlação de Spearman $(\alpha=0,01)$.

O mesmo acontece com a mesorregião Centro Ocidental e Metropolitana, com exceção das correlações com a variável C6 que são de moderadas e significativas $(a=0,05)$. Nas mesorregiões Centro Oriental, Nordeste, Sudeste e Sudoeste a maioria das correlações são fortes e significativas $(\alpha=0,01)$, com exceção da variável C6 que apresenta correlações fracas a muito fracas e não significativas.

Resultados semelhantes foram encontrados por Nascimento et al. (2012) que encontraram as variáveis: BP, C6, M, F sendo significativas e as variáveis PV, PC, DG, MIR diferentemente do presente estudo foram não significativas em relação ao número de óbitos infantis. Os resultados encontrados para a variável C6 nas mesorregiões Centro Oriental, Sudoeste e Sudeste corroboram com os estudos realizado por Santos et al. (2012) e Brum et al. (2014), que usaram análise bivariada e teste qui-quadrado de associação respectivamente para verificar a associação entre óbitos infantis e a variável C6. Resultados adversos foram encontrados Geib et al. (2010) para a mesma variável.

Conforme Paim et al. (2011), desde 2007-2008, o número insuficiente de visitas pré-natal, já não eram um preditor da mortalidade infantil no Brasil. Melhorias no campo das políticas sociais e cuidados primários são possíveis explicações para esta conclusão. 
Tabela 1- Coeficiente de Correlação e Significância do Teste de Correlação de Spearman do número de óbitos infantis no Rio Grande do Sul e mesorregiões e das demais variáveis

\begin{tabular}{|c|c|c|c|c|c|c|c|c|}
\hline Variáveis & $\begin{array}{c}\text { Rio } \\
\text { Grande } \\
\text { do Sul }\end{array}$ & $\begin{array}{l}\text { Centro } \\
\text { Ocidental }\end{array}$ & $\begin{array}{l}\text { Centro } \\
\text { Oriental }\end{array}$ & Metropolitana & Nordeste & Noroeste & Sudeste & Sudoeste \\
\hline $\mathrm{BP}$ & $0,9320 * \star$ & $0,8629 * \star$ & $0,9396 * *$ & $0,9672 * *$ & $0,9289 * *$ & $0,8593 * *$ & $0,9712^{* *}$ & $0,9938 * *$ \\
\hline DG & 0,9276 ** & $0,8676 * *$ & 0,9591 ** & $0,9671 * *$ & $0,8755^{* *}$ & $0,8511 * *$ & $0,9683^{* *}$ & $0,9860 * *$ \\
\hline MIR & $0,9909 * *$ & $0,9777 * *$ & $0,9877 * *$ & $0,9937 * *$ & $0,9915^{* *}$ & 0,9561 ** & $0,9983 * *$ & $0,9995 * *$ \\
\hline PV & $0,8788 * *$ & $0,8689 * *$ & $0,9146 * *$ & $0,9351 * *$ & $0,8816 * *$ & $0,7716 * *$ & $0,8808 * *$ & $0,9808 * *$ \\
\hline PC & $0,9173^{* *}$ & $0,9241 * *$ & $0,9522 * *$ & $0,9337 * *$ & $0,9103 * *$ & $0,8502 * *$ & $0,9029 * *$ & $0,9710 * *$ \\
\hline C6 & $0,3510 * *$ & $0,4281 *$ & $0,2666^{\mathrm{ns}}$ & $0,4445^{\star}$ & $0,2487^{n s}$ & $0,1848 * *$ & $0,0543^{n s}$ & $0,1086^{\mathrm{ns}}$ \\
\hline$M$ & $0,9246 * *$ & $0,7890 * *$ & $0,9494 * *$ & $0,9681 * *$ & $0,9290 * *$ & $0,8566 * \star$ & $0,9566 * *$ & $0,9845 * *$ \\
\hline $\mathrm{F}$ & $0,8734 * *$ & $0,9197 * *$ & $0,9097 * *$ & $0,9367 * *$ & $0,8627 * *$ & 0,7654 ** & $0,9706 * *$ & 0,9611 ** \\
\hline S1 & $0,9125 * *$ & $0,8394 * *$ & $0,9306 * *$ & $0,9388 * *$ & $0,8834 * *$ & $0,8757 * *$ & $0,9587 * \star$ & $0,9886 * *$ \\
\hline
\end{tabular}

\subsection{Análise de Componentes Principais}

Nas Tabelas 2 a 9 são apresentados os autovalores, a proporção de explicação individual e a proporção de explicação acumulada de cada componente principal. A Análise de Componentes Principais revela que tanto o Rio Grande do Sul (Tabela 2) quanto suas mesorregiões (Tabelas 3 a 9) têm as variáveis explicadas por apenas duas componentes, pois as mesmas explicam conjuntamente, em todas as situações, mais de $99 \%$. A primeira componente conta com a agregação das variáveis óbitos infantis, baixo peso ao nascer, duração gestacional, mulheres em idade reprodutiva, tipo de parto, sexo do bebê e óbitos ocorridos na primeira semana de vida, sendo mais explicativa que a segunda, tanto no estado quanto nas mesorregiões, explicando quase $90 \%$ da variação total das variáveis originais.

A segunda componente conta apenas com a variável proporção de mulheres que realizaram menos de 7 consultas pré-natais, explicando aproximadamente 10\% da variabilidade. Os gráficos Scree-plot (Figuras 1 a 8) apontam a importância de cada componente principal para o estado e suas mesorregiões. Para o caso da Figura 1, o primeiro autovalor representa o número de variáveis explicadas pela primeira componente, desta forma, o autovalor de 8,9799 (Tabela 2) explica a variância unitária de 9 variáveis. Já o segundo autovalor, é necessário para explicar a variável C6. Esse mesmo comportamento pode ser visto nas demais figuras e tabelas. 
O Quadro 2 apresenta as correlações das duas componentes principais com as variáveis originais. Corroborando com o mencionado anteriormente, a primeira componente apresenta correlações elevadas com 9 variáveis (OI, BP, DG, MIR, PC, PV, F, M, S1). A variável C6 apresentou correlação baixa com a primeira componente e alta com a segunda, indicando a importância da segunda componente.

Nascimento et al.(2012) confirmam que o baixo peso ao nascer e o sexo masculino do bebê são fatores importantes para o número de óbitos infantis. Geib et al. (2010), Loose et al.(2014) também encontraram em suas pesquisas a variável baixo peso ao nascer como relevante. Estes resultados também foram encontrados neste artigo para o estado do Rio Grande do Sul e suas respectivas mesorregiões.

Santos et al. (2012) encontraram a variável proporção de mulheres que realizaram menos de 7 consultas pré-natais como importante no número de óbitos infantis corroborando com o presente artigo. E ao contrário, os autores depararam o parto cesariano como não importante.

Tabela 2 - Porcentagem de variação explicada pelos componentes: Rio Grande do Sul

\begin{tabular}{lccc}
\hline & Componente1 & Componente 2 & Componente 3 \\
\hline OI & $-0,3336$ & 0,0193 & $-0,0326$ \\
BP & $-0,3331$ & 0,0317 & 0,0362 \\
DG & $-0,3331$ & 0,0317 & 0,0794 \\
MIR & $-0,3335$ & 0,0228 & $-0,0796$ \\
PV & $-0,3308$ & 0,0084 & $-0,7722$ \\
PC & $-0,3319$ & 0,0305 & 0,5592 \\
C6 & $-0,0663$ & $-0,9975$ & 0,0147 \\
M & $-0,3328$ & 0,021 & $-0,1112$ \\
F & $-0,3324$ & 0,017 & 0,0748 \\
S1 & $-0,3323$ & 0,0163 & 0,2404 \\
Autovalor & 8,9799 & 0,9652 & 0,029 \\
\% variância & 89,7986 & 9,6523 & 0,2903 \\
\% da variância acumulada & 89,7986 & 99,4509 & 99,7412 \\
\hline
\end{tabular}


Figura 1 - Scree-plot das variâncias dos componentes: Rio Grande do Sul

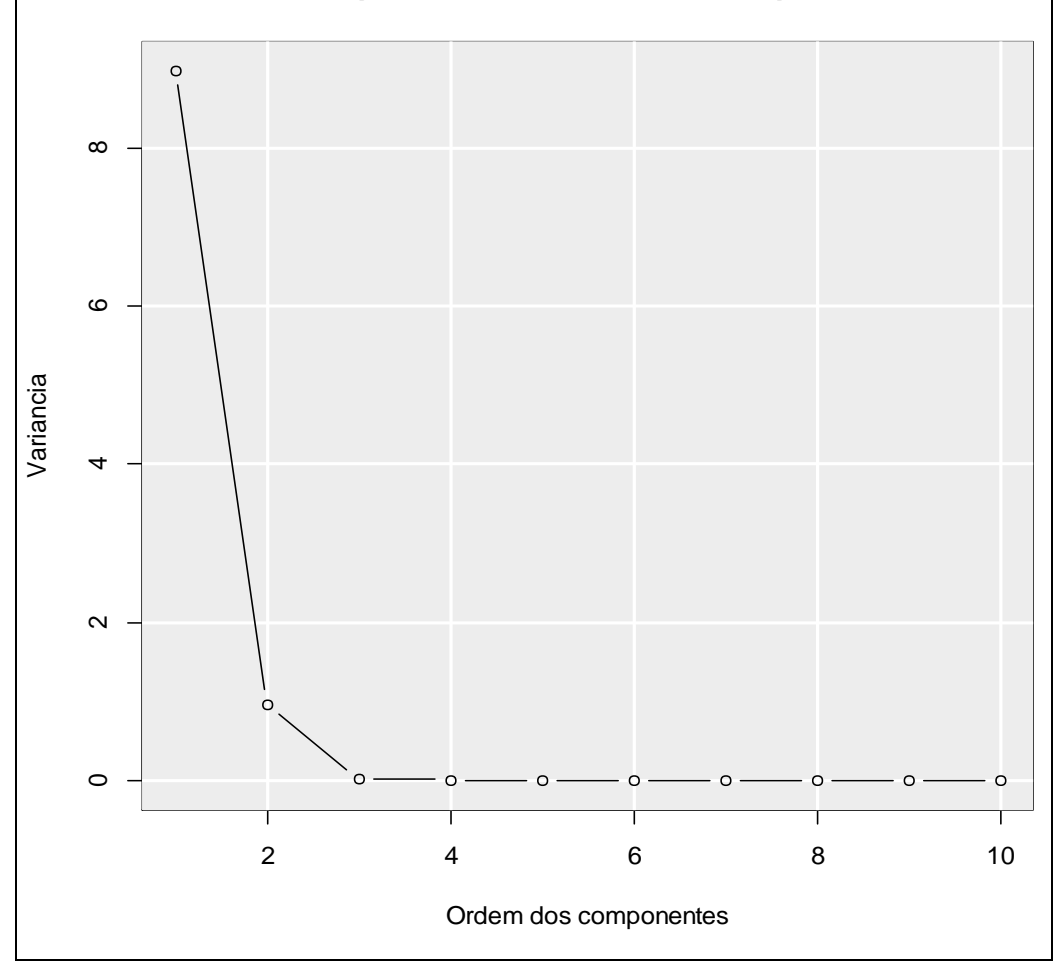

Tabela 3 - Porcentagem de variação explicada pelos componentes: Centro Oriental

\begin{tabular}{lccc}
\hline & Componente1 & Componente 2 & Componente 3 \\
\hline OI & $-0,3361$ & 0,0214 & 0,0609 \\
BP & $-0,3343$ & 0,0335 & $-0,09$ \\
DG & $-0,3344$ & 0,0313 & $-0,0205$ \\
MIR & $-0,3354$ & 0,0195 & 0,1516 \\
PV & $-0,3317$ & 0,0403 & $-0,044$ \\
PC & $-0,3325$ & 0,007 & 0,2187 \\
C6 & $-0,0668$ & $-0,9972$ & $-0,0028$ \\
M & $-0,3305$ & 0,0243 & $-0,4766$ \\
F & $-0,3282$ & 0,0167 & 0,6784 \\
S1 & $-0,3301$ & 0,0059 & $-0,477$ \\
Autovalor & 8,8275 & 0,9659 & 0,1014 \\
\% variância & 88,2751 & 9,6593 & 1,0143 \\
\% da variância acumulada & 88,2751 & 97,9345 & 98,9488 \\
\hline
\end{tabular}


Figura 2- Scree-plot das variâncias dos componentes: Centro Oriental

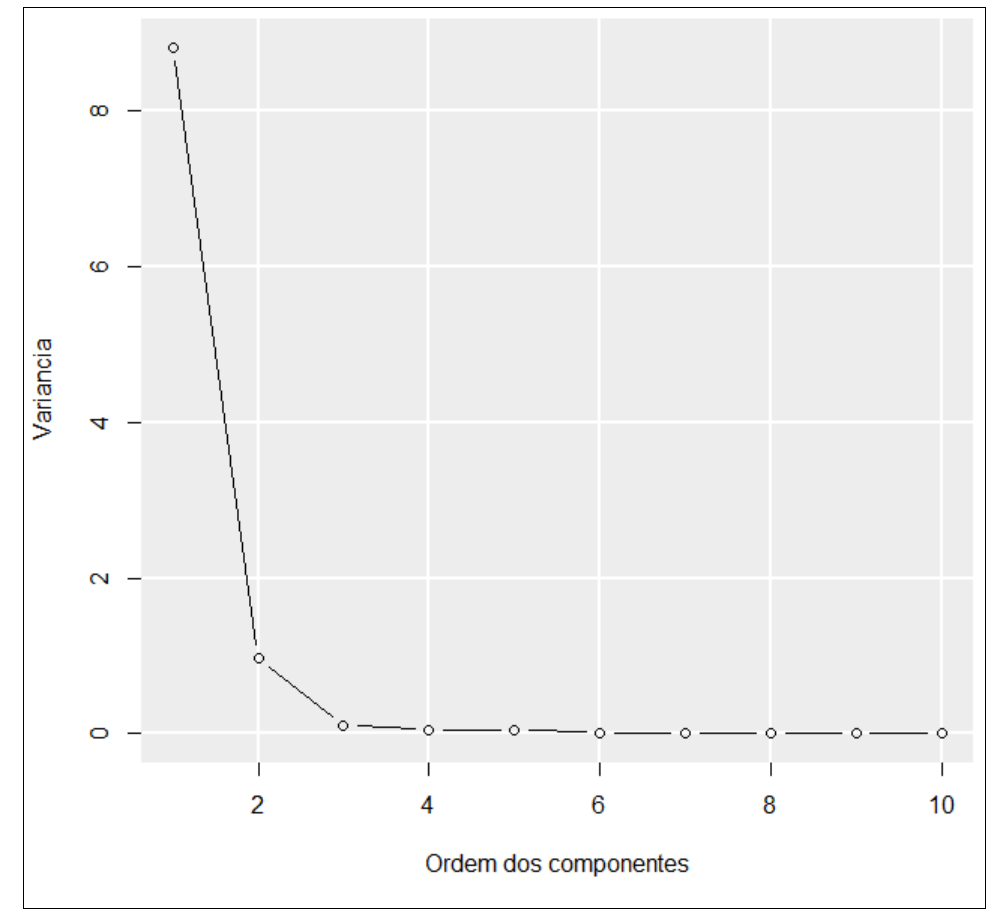

Tabela 4 - Porcentagem de variação explicada pelos componentes: Centro Ocidental

\begin{tabular}{lccc}
\hline & Componente1 & Componente 2 & Componente 3 \\
\hline OI & $-0,3335$ & $-0,0279$ & $-0,0079$ \\
BP & $-0,3329$ & $-0,0235$ & 0,178 \\
DG & $-0,3329$ & $-0,025$ & 0,0863 \\
MIR & $-0,3334$ & $-0,028$ & 0,0012 \\
PV & $-0,3319$ & $-0,015$ & 0,4749 \\
PC & $-0,3323$ & $-0,0341$ & $-0,3588$ \\
C6 & $-0,0812$ & 0,9965 & $-0,0078$ \\
M & $-0,3320$ & $-0,0237$ & $-0,3703$ \\
F & $-0,3309$ & $-0,0332$ & 0,4831 \\
S1 & $-0,3303$ & $-0,0331$ & $-0,4857$ \\
Autovalor & 8,9836 & 0,9473 & 0,0333 \\
\% variância & 89,8362 & 9,4732 & 0,3334 \\
\% da variância acumulada & 89,8362 & 99,3094 & 99,6428 \\
\hline
\end{tabular}


Figura 3 - Scree-plot das variâncias dos componentes: Centro Ocidental

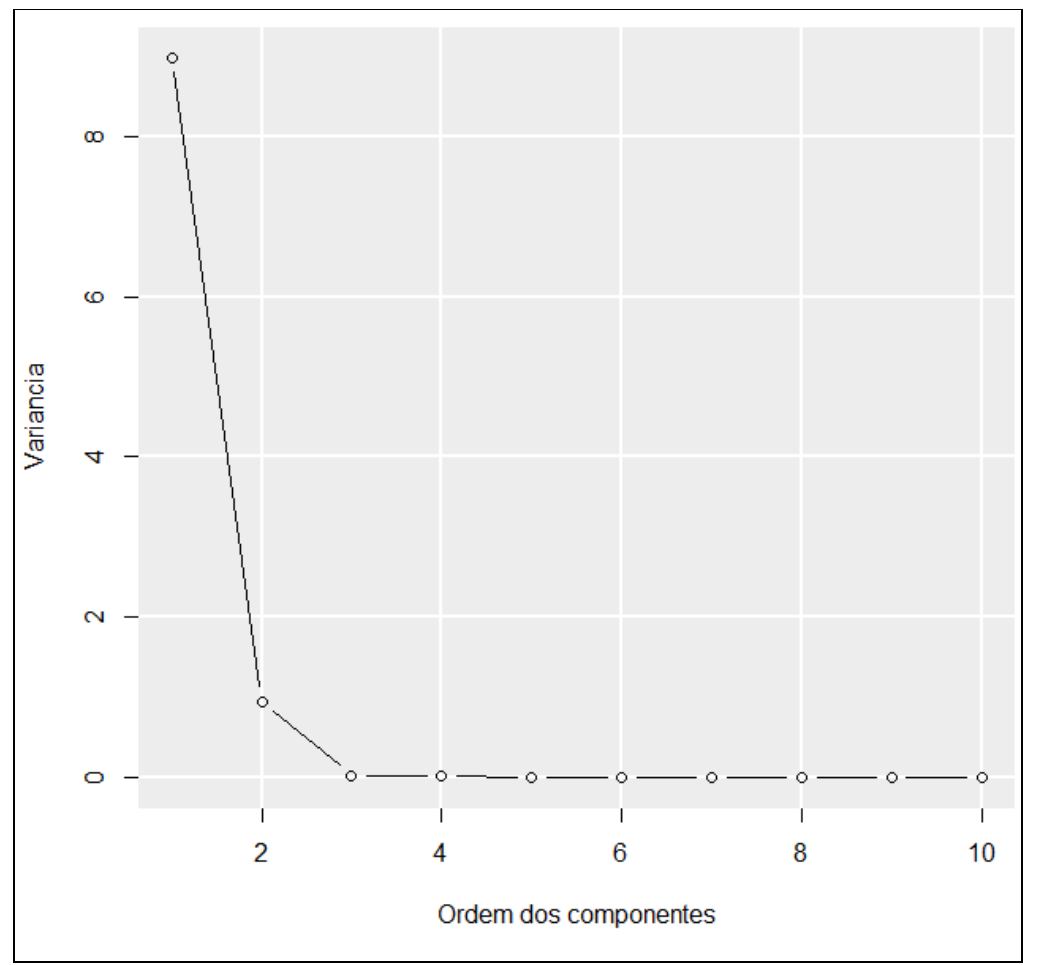

Tabela 5 - Porcentagem de variação explicada pelos componentes: Metropolitana

\begin{tabular}{lccc}
\hline & Componente1 & Componente 2 & Componente 3 \\
\hline OI & -0.3326 & -0.0224 & -0.0037 \\
BP & -0.3323 & -0.0400 & 0.1012 \\
DG & -0.3324 & -0.0361 & 0.1041 \\
MIR & -0.3326 & -0.0271 & 0.0735 \\
PV & -0.3320 & -0.0231 & 0.5015 \\
PC & -0.3322 & -0.0238 & -0.4003 \\
C6 & -0.0812 & 0.9965 & 0.0078 \\
M & -0.3321 & -0.0272 & 0.4358 \\
F & -0.3319 & -0.0148 & -0.5489 \\
S1 & -0.3319 & -0.0289 & -0.2655 \\
Autovalor & 9,0316 & 0,9471 & 0,0096 \\
\% variância & 90,3165 & 9,4715 & 0,0964 \\
\% da variância acumulada & 90,3165 & 99,7879 & 99,8844 \\
\hline
\end{tabular}


Figura 4 - Scree-plot das variâncias dos componentes: Metropolitana

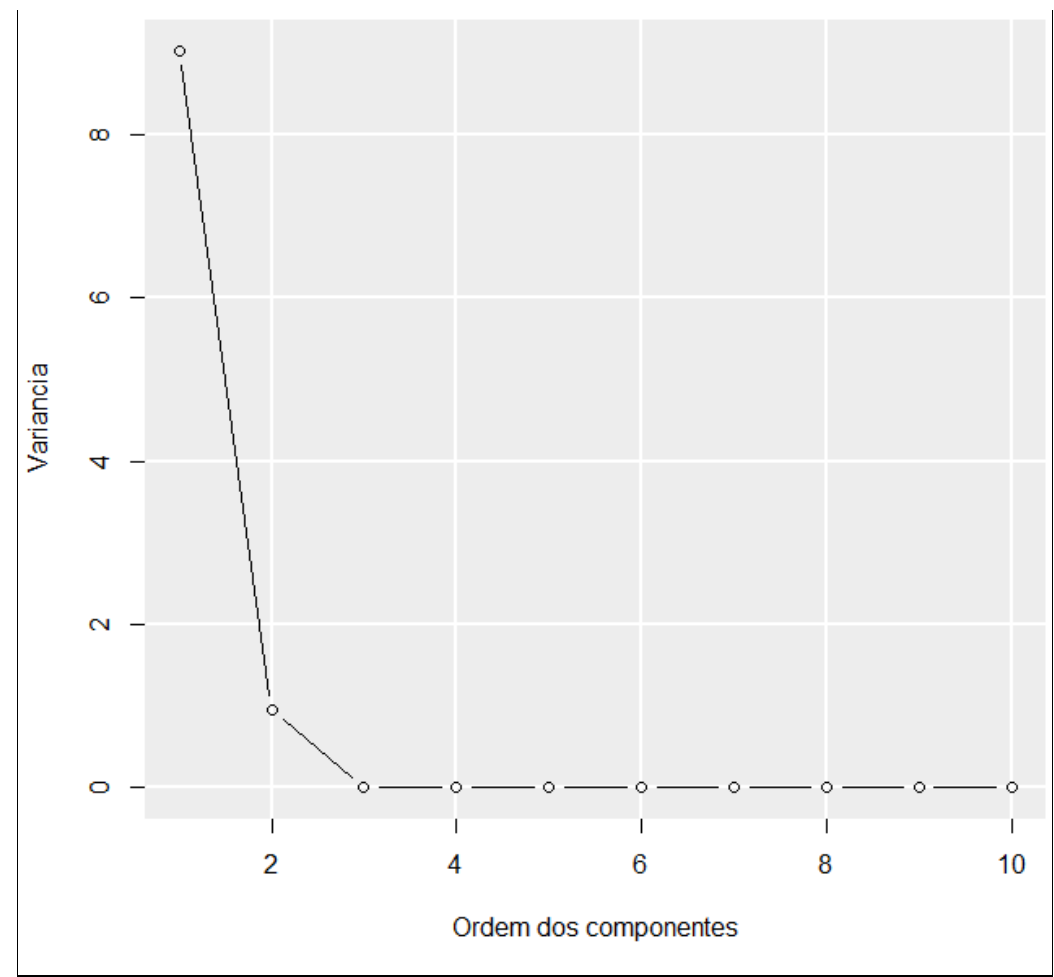

Tabela 6 - Porcentagem de variação explicada pelos componentes: Nordeste

\begin{tabular}{lccc}
\hline & Componente1 & Componente 2 & Componente 3 \\
\hline OI & -0.3338 & 0.0005 & -0.0421 \\
BP & -0.3336 & 0.0093 & 0.0736 \\
DG & -0.3335 & 0.0135 & 0.1650 \\
MIR & -0.3338 & 0.0006 & 0.0200 \\
PV & -0.3319 & -0.0161 & -0.7562 \\
PC & -0.3332 & 0.0098 & 0.4143 \\
C6 & -0.0093 & -0.9996 & 0.0253 \\
M & -0.3334 & 0.0076 & 0.2419 \\
F & -0.3332 & -0.0068 & -0.3389 \\
S1 & -0.3334 & 0.0096 & 0.2182 \\
Autovalor & 8,9720 & 1,0000 & 0,0190 \\
\% variância & 89,7201 & 10,0002 & 0,1903 \\
\% da variância acumulada & 89,7201 & 99,7203 & 99,9106 \\
\hline
\end{tabular}


Figura 5 - Scree-plot das variâncias dos componentes: Nordeste

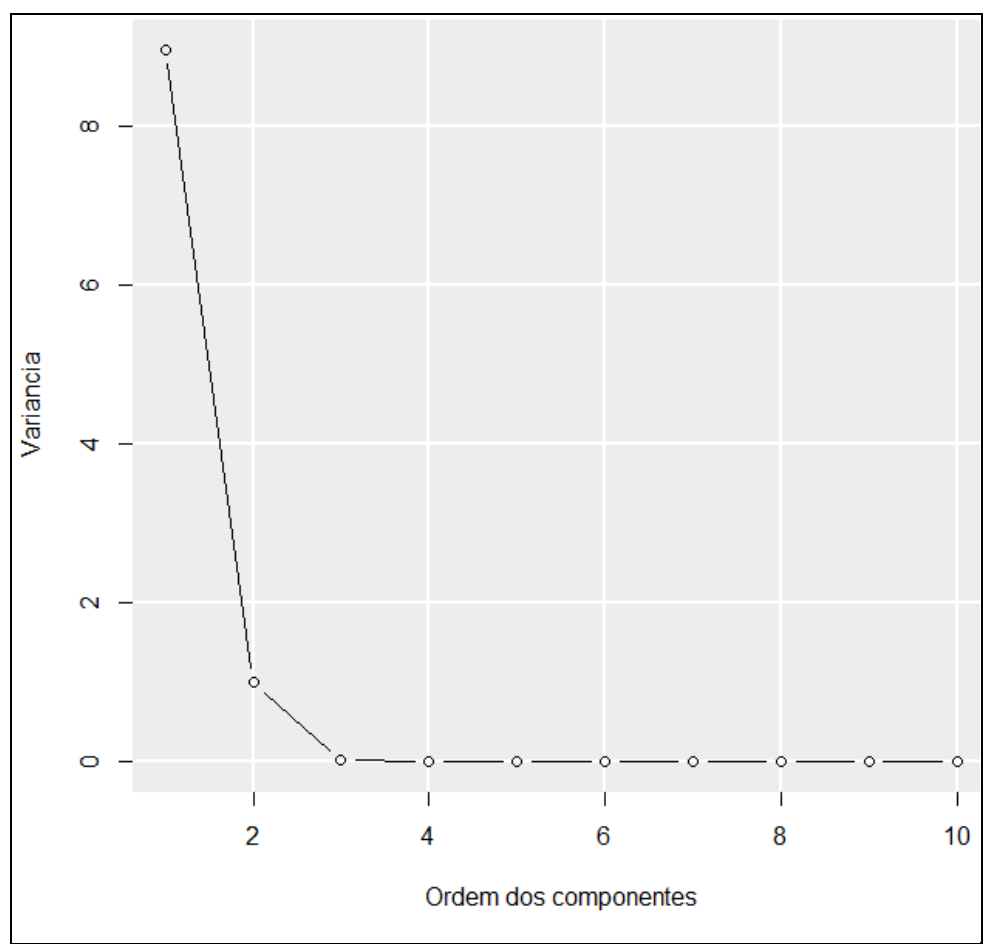

Tabela 7 - Porcentagem de variação explicada pelos componentes: Noroeste

\begin{tabular}{lccc}
\hline & Componente1 & Componente 2 & Componente 3 \\
\hline OI & -0.3356 & 0.0132 & 0.0182 \\
BP & -0.3341 & 0.0332 & 0.1109 \\
DG & -0.3334 & 0.0438 & 0.0499 \\
MIR & -0.3355 & 0.0245 & -0.0206 \\
PV & -0.3276 & -0.0149 & -0.7716 \\
PC & -0.3316 & 0.0476 & 0.5024 \\
C6 & -0.0697 & -0.9961 & 0.0432 \\
M & -0.3311 & 0.0135 & -0.2380 \\
F & -0.3306 & 0.0116 & 0.2739 \\
S1 & -0.3331 & 0.0358 & 0.0557 \\
Autovalor & 8,8554 & 0,9644 & 0,0738 \\
\% variância & 88,5539 & 9,6438 & 0,7378 \\
\% da variância acumulada & 88,5539 & 98,1977 & 98,9355 \\
\hline
\end{tabular}


Figura 6 - Scree-plot das variâncias dos componentes: Noroeste

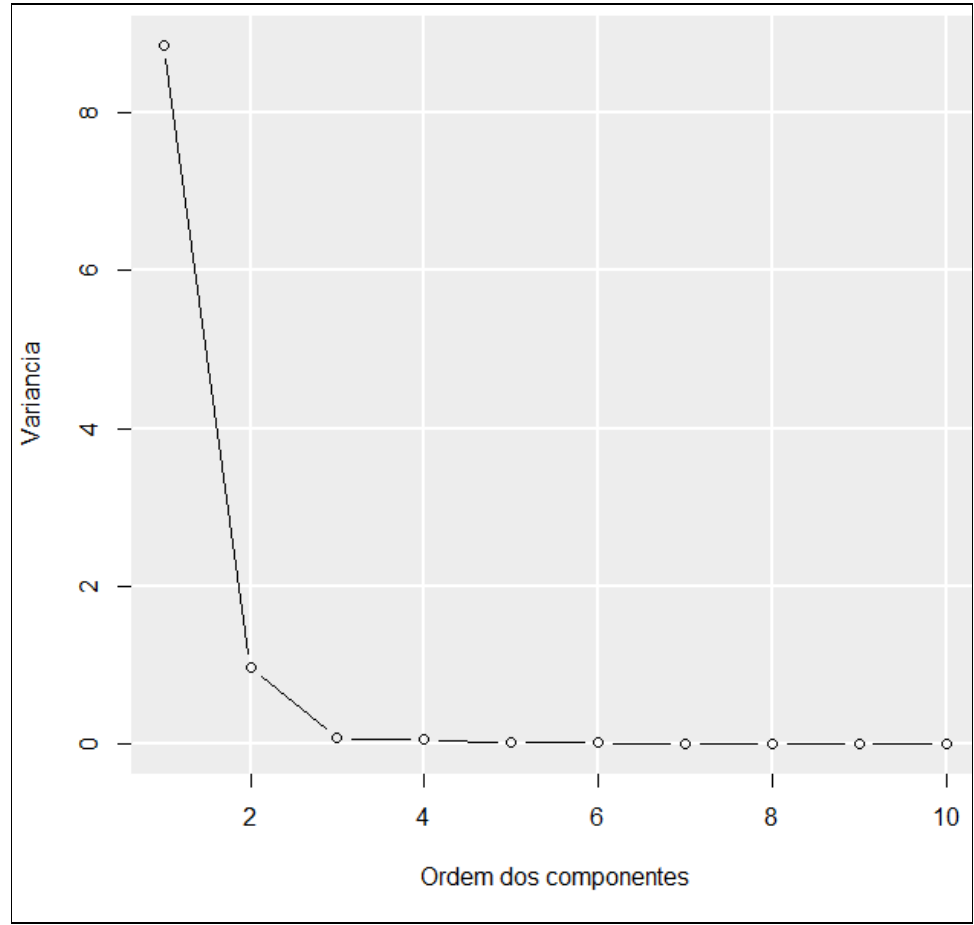

Tabela 8 - Porcentagem de variação explicada pelos componentes: Sudeste

\begin{tabular}{lccc}
\hline & Componente1 & Componente 2 & Componente 3 \\
\hline OI & -0.3353 & 0.0133 & -0.0194 \\
BP & -0.3348 & 0.0078 & 0.1679 \\
DG & -0.3345 & 0.0123 & 0.2089 \\
MIR & -0.3352 & 0.0101 & -0.0703 \\
PV & -0.3229 & 0.0160 & -0.8322 \\
PC & -0.3315 & 0.0186 & 0.4611 \\
C6 & -0.0363 & -0.9992 & -0.0026 \\
M & -0.3349 & 0.0119 & 0.0364 \\
F & -0.3345 & 0.0172 & -0.0780 \\
S1 & -0.3344 & 0.0021 & 0.1012 \\
Autovalor & 8,8897 & 0,9898 & 0,1054 \\
\% variância & 88,8965 & 9,8978 & 1,0540 \\
\% da variância acumulada & 88,8965 & 98,7943 & 99,8484 \\
\hline
\end{tabular}




\section{CONCLUSÃO}

Diante dos resultados obtidos, pode-se concluir de um modo geral, o cenário da mortalidade infantil no Rio Grande do Sul se reflete em suas mesorregiões. A correlação entre as variáveis a nível de estado se mostram fortes, com exceção das correlações com a variável C6. Este mesmo comportamento pode ser observado na maioria das mesorregiões estudadas, com exceção das mesorregiões Centro Oriental, Sudoeste e Sudeste onde ocorrem algumas correlações não significativas com a variável C6.

Com a análise de componentes principais, tanto no estado como em suas mesorregiões a primeira componente explica aproximadamente $90 \%$ das variações totais, a segunda componente explica aproximadamente 10\%. Assim, os variáveis óbitos infantis, baixo peso ao nascer, duração gestacional, mulheres em idade reprodutiva, tipo de parto, sexo do bebê, óbitos ocorridos na primeira semana de vida e proporção de mulheres que realizaram menos de 7consultas pré-natais podem ser consideradas variáveis importantes sobre a mortalidade infantil. 


\section{REFERÊNCIAS}

AHMAD O B, LOPEZ A D, INOUE M. The decline in child mortality: a reappraisal. Bull WHO. 2000; 78(10):1175-91.

ANDRADE SM, SOARES D A, MATSUO T, SOUZA R K T, MATHIAS T A F, IWAKURA M L H, Condições de vida e mortalidade infantil no Estado do Paraná, Brasil, 1997/2001. Cad. Saúde Pública. 2006; 22(1):181-189.

ARAÚJO BF, BOZZETTI MC, TANAKA AC. Mortalidade neonatal precoce no município de Caxias do Sul: um estudo de coorte. Jornal de Pediatria. 2000; 76(3):200-206.

BRASIL. Ministério da Saúde. Portaria n. 569, de $\mathbf{1}^{\circ}$ de junho de 2000. Estabelece o Programa de Humanização no Pré-natal e Nascimento. 2000.

BRUM C A, STEIN A T, PELLANDA L C. Mortalidade Infantil em Novo Hamburgo: Fatores Associados e Causas Cardiovasculares. Arquivos Brasileiros de Cardiologia. 2014.

CLAESON M, BOS E R, MAWJI T. Reducing child mortality in India in the new millennium. Bull WHO. 2000; 78(10):1192-1199.

DANCEY C, REIDY J. Estatística Sem Matemática para Psicologia: Usando SPSS para Windows. Porto Alegre, Artmed, 2005.

ESCOFIER B, PAGÈS J. Análisis factorialies simples y multiples: objetctivo, métodos y interpretación. Bilbao. Ed. Universidaddel Pais Vasco, 1992.

FERREIRA DF. Estatística Básica. 1 ed. Lavras: Editora UFLA: 2005.

FERREIRA DF. Estatística Multivariada. 2 ed. Lavras: Editora UFLA: 2011.

GEIB LTC, FRÉU C M, BRANDÃO M, NUNES M L. Determinantes sociais e biológicos da mortalidade infantil em coorte de base populacional em Passo Fundo, Rio Grande do Sul. Ciência \& Saúde Coletiva. 2010;15(2):363-370.

HERNANDEZ A R, SILVA H C, AGRANONIK M, QUADROS F M, GOLDANI M Z. Análise de tendências das taxas de mortalidade infantil e de seus fatores de risco na cidade de Porto Alegre, Rio Grande do Sul, Brasil,no período de 1996 a 2008. Cad. Saúde Pública, 2011; 27(11):2188-2196.

IBGE - INSTITUTO BRASILEIRO DE GEOGRAFIA E ESTATÍSTICA, Projeção da População do Brasil, 2013,

JOHSON R A, WICHERN D W. Applied multivariate statistical analysis. 3. ed. Englewood Cliffs: Prentice Hall, 1992. 
LOOSE L H, SILVA C P, JACOBI L F, MORAES A B. A utilização da curva ROC para identificar fatores de risco associados à mortalidade infantil. Revista Brasileira de Estatística. 2014; 75(24):7-25.

MENEZES AM, HALLAL PC, SANTOS I S, VICTORA C G, BARROS F, C. Infant mortality in Pelotas, Brazil: a comparison of risk factors in two birth cohorts. Rev Panam Salud Pública. 2005; 18(6):439-46.

MINISTÉRIO DA SAÚDE. Portal do DATASUS. Disponível em: http://www2.datasus.gov.br/DATASUS/index.php?area=0205. Acesso em: 10 de outubro de 2016.

MOITA NETO JM, MOITA GC. Uma introdução à análise exploratória de dados multivariados. Química Nova. 1998; 21(4): 467-469.

NASCIMENTO R M, LEITE AJ M, ALMEIDA N MGD, ALMEIDA PC, SILVA CF. Determinantes da mortalidade neonatal: estudo caso-controle em Fortaleza, Ceará, Brasil. Caderno de Saúde Pública. 2012;28(3):559-572.

NUNES A D S, AMADOR AE, DANTAS APQ M, AZEVEDO UM, BARBOSA IR. Acesso à assistência pré-natal no Brasil: análise dos dados da pesquisa nacional de saúde. Revista Brasileira em Promoção da Saúde. 2017; 30(3): 1-10.

OLIVEIRA L, MENDES M. Mortalidade infantil no Brasil: uma avaliação de tendências recentes. In: Minayo. Os muitos Brasis - saúde e população na década de $\mathbf{8 0}$. São Paulo: ABRASCO, 1990; 24(1): 291-303.

ONU - ORGANIZAÇÃO DAS NAÇÕES UNIDAS, Levels \& Trends in Child Mortality: Estimates Developed by the UN Inter-agency Group for Child Mortality Estimation United, 2015.

PAIM J, TRAVASSOS C, ALMEIDA C, BAHIA L, MACINKOJ. The Brazilian health system: history, advances, and challenges. Lancet. 2011;377(9779):1778-1797.

R DEVELOPMENT CORE TEAM (2015).R:a language and environment for Statistical computing. Disponível em: http://www.R-project.org/. Acesso em: 12 março de 2010.

SANTOS H G, ANDRADE SM, SILVA AMR, CARVALHO WO, MESAS AE. Risk factors for infant mortality in a municipality in southern Brazil: a comparison of two cohorts using hierarchical analysis. Caderno Saúde Púbica. 2012;28(10):1915-1926.

SCALON, J. D.; JARDIM, S. S. L.; SANTOS, G. R.; NOGUEIRA, D. A. Análise do padrão espacial da mortalidade infantil utilizando geoestatística. Revista UniVap. 2012;18(32):149-160. 
SHIMAKURA SE, CARVALHO M S, AERTS DRGC, FLORES R. Distribuição espacial do risco: modelagem da mortalidade infantil em Porto Alegre, Rio Grande do Sul, Brasil. Caderno de Saúde Pública. 2001;17(5):1251-1261.

VERMELHO LL, LEAL AJC, KALE P L. Indicadores de Saúde, In: Medronho, Epidemiologia, São Paulo: Atheneu, 2006. 